Research Paper

\title{
Curative Resection of Hepatocellualr Carcinoma Using Modified Glissonean Pedicle Transection versus the Pringle Maneuver: A Case Control Study
}

\author{
Bai Ji*, Yingchao Wang*, Guangyi Wang, Yahui Liu ${ }^{\bowtie}$ \\ Department of Hepatobiliary and Pancreatic Surgery, the First Hospital, Jilin University, Jilin 130021, China. \\ * Bai Ji and Yingchao Wang contributed equally to this manuscript.
}

$\triangle$ Corresponding author: Yahui Liu, PhD. MD, Department of Hepatobiliary and Pancreatic Surgery, the First Hospital, Jilin University, Jilin 130021, China (Tel: 86-431-88782421; E-mail:liuyahui2008@yeah.net).

(c) Ivyspring International Publisher. This is an open-access article distributed under the terms of the Creative Commons License (http://creativecommons.org/ licenses/by-nc-nd/3.0/). Reproduction is permitted for personal, noncommercial use, provided that the article is in whole, unmodified, and properly cited.

Received: 2012.07.14; Accepted: 2012.10.15; Published: 2012.11.01

\begin{abstract}
Objective: The Glissonean pedicle transection method of liver resection has been found to shorten operative time and minimize intraoperative bleeding during liver segmentectomy. We have compared the feasibility, effectiveness, and safety of the Glissonean pedicle transection method with the Pringle maneuver in patients undergoing selective curative resection of large hepatocellualr carcinoma (HCC).

Methods: Eligible patients with large $(>5 \mathrm{~cm})$ nodular HCC $(\mathrm{n}=50)$ were assigned to undergo curative hepatectomy using the Glissonean pedicle transection method $(n=25)$ or the Pringle maneuver $(n=25)$. Partial interruption of the infrahepatic inferior vena cava was incorporated to further reduce bleeding from liver transection. The primary outcome measure was postoperative changes in liver function from baseline. Secondary outcomes included operating time, volume of intraoperative blood loss/transfusion, and time to resolution of ascites.

Results: The two groups were comparable in age, sex, site and size of the liver tumor, segment or lobe intended to be resected, and liver function reserve, and the results were not significant statistically. All patients underwent successful major hepatectomies using the assigned method, with the extent of major hepatectomy comparable in the two groups $(P=$ 0.832 ). The Glissonean approach was associated with shorter hepatic inflow interruption $(30.0 \pm 12.0 \mathrm{~min}$ vs. $45.0 \pm 13.0 \mathrm{~min}, P<0.00 \mathrm{I})$, lower volume of blood loss $(\mid 45.0 \pm 20.0 \mathrm{~mL}$ vs. $298.0 \pm 109.0 \mathrm{~mL}, P<0.00 \mathrm{I})$, reduced requirement for transfusion $(0.0 \pm 0.0 \mathrm{~mL}$ vs. 200.0 $\pm 109.0 \mathrm{~mL}, P<0.000 \mathrm{I})$, and more rapid resolution of ascites $(9.5 \pm 1.2 \mathrm{~d}$ vs. $15.3 \pm 2.4 \mathrm{~d}, P<$ $0.001)$. Postoperative liver function measures were comparable in the two groups, and the results were not significant statistically.

Conclusion: The Glissonean pedicle transection method is a feasible, effective, and safe technique for hepatic inflow control during the curative resection of large nodular HCCs.
\end{abstract}

Key words: Hepatocellualr carcinoma, large nodular; Pringle maneuver; Glissonean pedicel transection; Partial interruption of inferior vena cava; Surgical outcomes.

\section{Introduction}

Chinese patients have the highest HCC morbidity and mortality rates in the world, with over $75 \%$ of these patients having large nodular HCCs (diameter $\geq$ $5 \mathrm{~cm})^{1}$. The management of HCC is a huge challenge 
due to the complicating background liver disease and the high rate of recurrence ${ }^{2}$. Many adjuvant modalities have been developed ${ }^{3}$. However, radical resection remains the most effective treatment of HCC in operable or potentially operable patients ${ }^{4}$. Although this treatment is associated with optimal prognosis, as shown by long-term survival rates, only $10-15 \%$ of patients with HCC are indicated for surgical resection $^{5}$. Historically, major hepatectomy for the treatment of HCC is highly risky and frequently accompanied by surgical morbidities, especially in patients with pre-existing liver function impairment 6 . The remnant liver volume should be no less than $25 \%$ of the total liver volume in non-cirrhotic patients, but should be greater than $40 \%$ of the total volume in cirrhotic subjects ${ }^{7}$.

Two of the determinants of surgical morbidity and patient outcome are massive bleeding during liver resection and the consequent need for blood transfusion ${ }^{8}$. Large-volume $(\geq 800 \mathrm{~mL})$ blood loss during resection has been reported to be an independent risk factor for postoperative morbidity, including liver dysfunction, in HCC patients with underlying liver diseases ${ }^{9}$. In addition, intraoperative transfusion has been reported associated with unfavorable prognosis in HCC patients undergoing hepatic resection, especially in patients with huge $(\geq 10$ $\mathrm{cm})$ liver tumors ${ }^{10}$. Fortunately, the survival outcomes of East Asian patients undergoing radical resection for HCC have shown continuous improvement $^{11}$.

The Pringle maneuver is the standard technique used to control liver bleeding in patients undergoing hepatectomy and those with emergency liver trauma 12. The primary disadvantage of this technique is the resultant ischemia-reperfusion injury, especially in cirrhotic livers less tolerant of anoxia and serum deprivation ${ }^{13}$. The Pringle maneuver is also associated with high risks of bacterial translocation, intrahepatic metastases, impaired liver regeneration, systemic inflammation, and multiple organ dysfunction syndrome 14. In addition, this maneuver cannot stop bleeding from the backflow of the hepatic veins or from the inferior vena cava ${ }^{15}$. This technique has been modified to control hilar blood flow, with measures including intermittent (15 min or $30 \mathrm{~min}$ ) occlusion, hemihepatic artery control (half-Pringle maneuver), and selective hepatic vascular exclusion for tumors involving the hepatic veins ${ }^{16}$. In addition to ischemic preconditioning, agents tested in preclinical and clinical studies include dexamethasone, dimethyl sulphoxide, somatostatin, ursodeoxycholic acid, protease inhibitor, and iNOS inhibitor ${ }^{17}$.

The Glissonean pedicle transection method is a new concept of liver segmentectomy based on Glissonean pedicle tree anatomy ${ }^{18}$. The liver can be divided into three segments, left, middle, and right, as well as a caudate area, in relation to the Glissonean pedicle tree. Each segment is fed by secondary branches of the Glissonean pedicle, while the caudate area is nourished by the primary branch directly. In liver resection using the Glissonean pedicle transection method, the vessels supplying the target segment are completely ligated and divided at the hepatic hilum prior to resection without exposing the hepatic artery, portal vein, and bile duct individually. This method simplifies the procedure of liver resection, shortens operation time, and, more importantly, minimizes intraoperative bleeding. Furthermore, the use of an endolinear stapling device shortens the time required for pedicle control and expedites subsequent liver resection. This approach has been also incorporated into laparoscopic anatomical hepatectomy, showing a good feasibility and safety profile ${ }^{19}$. More promisingly, a retrospective study showed that, relative to the conventional maneuver, the Glissonean method improved the 5-year survival rates of patients undergoing hepatectomy. Since the Glissonean method also significantly reduces, diffuse-type recurrence, this method may prevent intrahepatic metastases.

\section{Materials and Methods}

\section{Patient enrollment and assessment}

The study protocol was approved by the Institutional Review Board of the First Hospital of the Norman Bethune School of Medicine at Jilin University. Patients $(n=98)$ radiologically diagnosed with resectable primary HCC (nodular type, diameter $>5$ $\mathrm{cm}$ ) and scheduled for radical hepatectomy via laparotomy were consecutively enrolled between January 2008 and May 2011. All patients underwent routine hematological, biochemical, virological serology (HBV and HCV), and serum tumor marker (alpha fetoprotein) testing at baseline screening. Chest $\mathrm{X}$-rays and abdominal contrast-enhanced computed tomography scan or magnetic resonance imaging were performed to characterize the size, location, and anatomical relation of each liver tumor and to exclude any patients with radiologically detectable extrahepatic metastases. Serum albumin, total bilirubin, alanine aminotransferase (ALT), and aspartate aminotransferase (AST) concentrations and prothrombin time were measured to determine the Child-Pugh score. HCC patients were eligible for radical hepatectomy via the Pringle maneuver or Glissonean pedicle transection if their preoperative indocyanine 
green retention rate at 15 minutes (ICG R15) was less than $10 \%$ and they preservation of at least $30 \%$ of total liver volume was expected following resection. Patients were excluded if they had extrahepatic metastases, pre-existing serious cardiovascular or cerebrovascular disease, or renal dysfunction. In addition, Patient exclusion criteria include those with tumor thrombi extending to portal bifurcation. Tumor thrombi in this area will be squeezed to other part of the liver in Glissonian approach method20. No patient exhibited ascites on preoperative abdominal ultrasonography or computed tomography. All patients provided written informed consent prior to surgery and were blinded to the method used for hilar vascular control. None of these patients received any neoadjuvant therapy. Following baseline screening, matched patients with comparable baseline characteristics were randomized 1:1 to undergo major hepatectomy using the Glissonean pedicle transection method $(n=25)$ or the Pringle maneuver $(n=25)$. All the operations as well as perioperative management were performed by a specifically designated surgical team led by a senior hepatobiliary surgeon (corresponding author) and containing resident surgeons, anesthesiologists, radiologists, pathologists, and surgical nurses. The diagnostic and management protocols complied with the updated NCCN guidelines for $\mathrm{HCC}$ and were approved by the Institute and the local healthcare authority. If volume of blood loss intraoperatively blood transfusion should be considered.

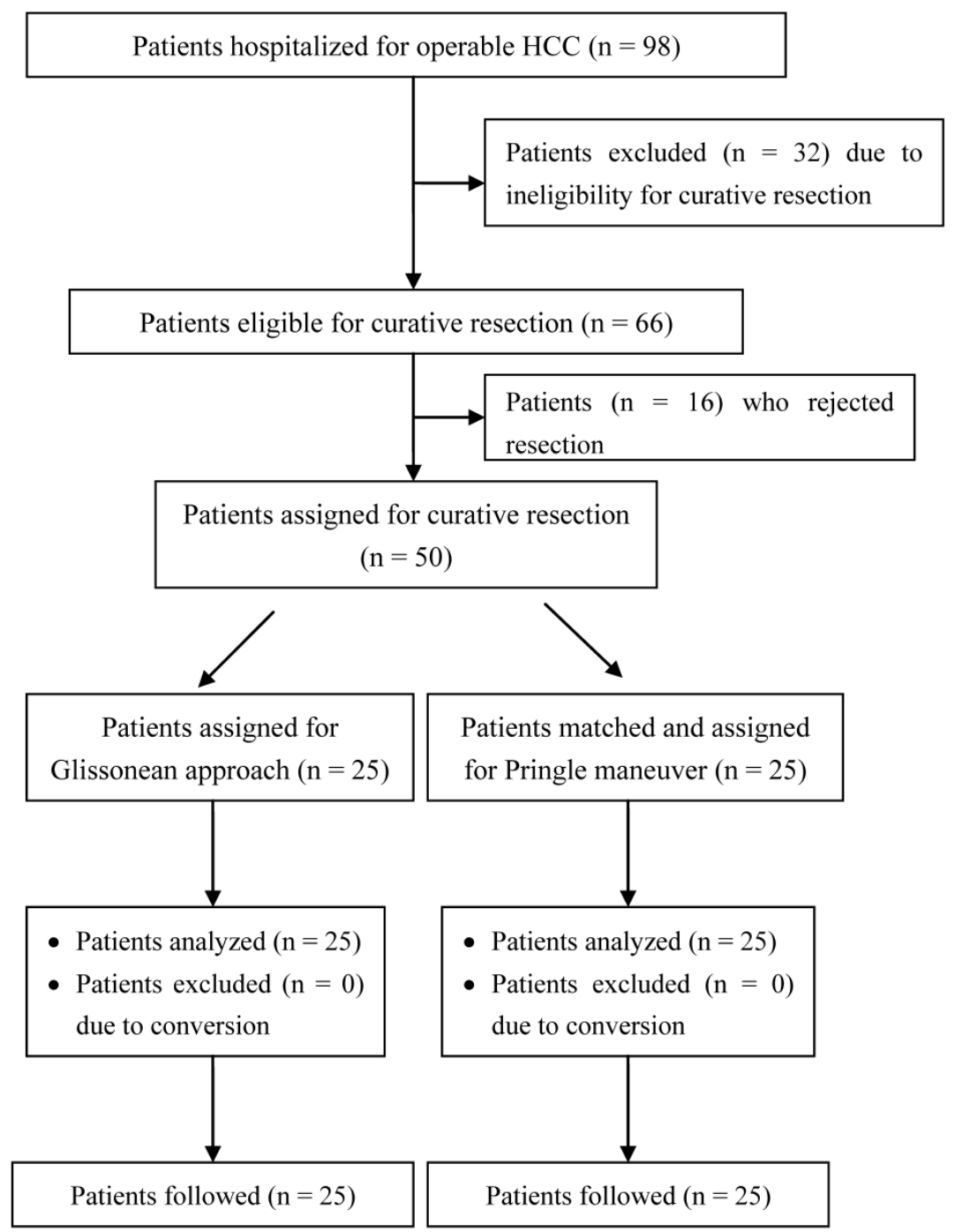

Figure I. Patient assignment flow chart. 


\section{Major hepatectomy using the Glissonean ped- icle transection method}

A laparotomy was performed through a bilateral subcostal incision. Peritoneal exploration was performed to reaffirm the resectability of the liver tumor and to exclude the presence of extrahepatic metastases and peritoneal seeding. Following liver mobilization, intraoperative ultrasonography was used to localize and characterize the liver tumor, including the number and sizes of tumors and their relationship to the vasculature. Cholecystectomy preceded the interruption of the hepatic hilum using a tourniquet. The hilar plate was dissected using Peng's multifunctional ('curettage and aspiration') operative dissector (PMOD; Shuyou Electric Science Co., Ltd., Hangzhou, China). The minor communicating vessels located inside the hilar plate were secured by cauterization. The hilar plate was further dissected downwards using a tangential clamp until reaching the posterior serosa of the hepatoduodenal ligament. The minor branches that flowed into the caudate area were appropriately preserved. In patients undergoing right or hemihepatectomy, the right or left hemihepatic pedicle, respectively was ligated using non-absorbable silk sutures (Fig. 2A); in patients undergoing right posterior lobectomy, the hepatic pedicles supplying the right posterior and anterior lobes were mobilized individually. The pedicle of the right posterior lobe was ligated with non-absorbable silk sutures, whereas the pedicle of the right anterior lobe was temporarily interrupted using an 8 F Foley catheter. The serosal membrane covering the IVC was dissected above the renal veins and below the lower liver margin using a tangential clamp. The infrahepatic IVC was encircled using an 8F Foley catheter and appropriately fastened. The central venous pressure (CVP) was reduced to $3 \mathrm{~cm} \mathrm{H} 2 \mathrm{O}$ to stop the backflow of the hepatic veins into the lobe or segment intended to be removed. The liver parenchyme was transected using the PMOD along the ischemia line.

\section{Major hepatectomy using the Pringle maneu- ver}

The Pringle maneuver was performed as previously described ${ }^{21}$. Briefly, the hepatoduodenal ligament was encircled using an $8 \mathrm{~F}$ Foley catheter and retracted towards the left side (Fig. 2B). The infrahepatic IVC was exposed right, anterior, and inferior to the hepatoduodenal ligament, and was encircled using an $8 \mathrm{~F}$ Foley catheter above the renal veins. The hilum was intermittently interrupted for 5 minutes initially and for 10 minutes afterwards, followed by the interruption of the infrahepatic IVC at the same intervals. Liver transection was performed using the PMOD.
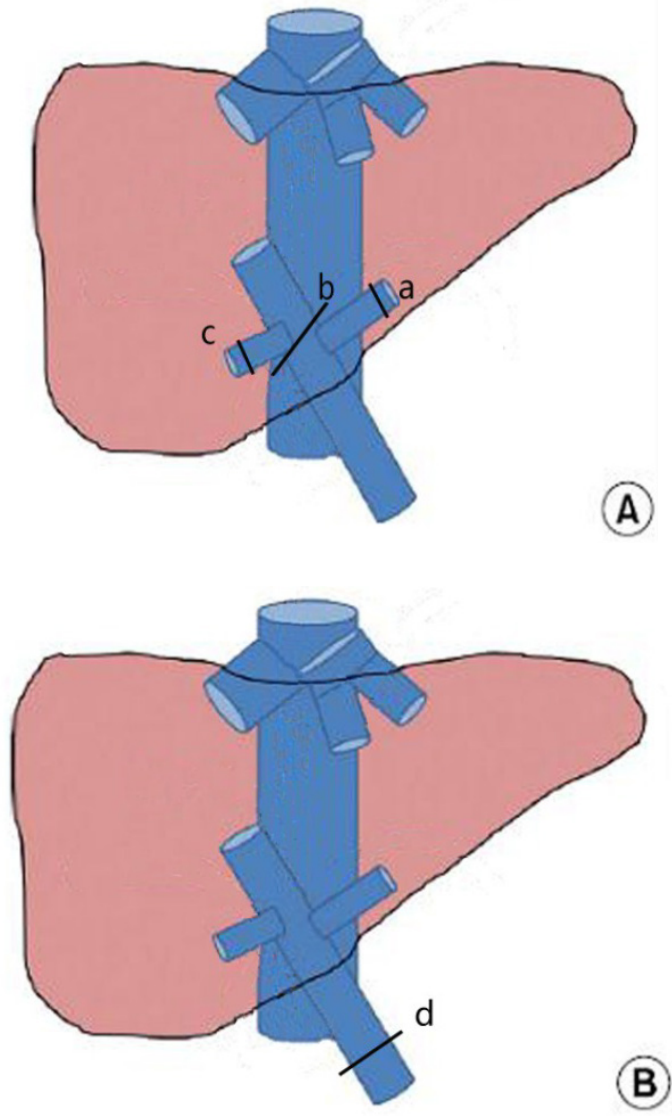

Figure 2. Hilar interruption using the Glissonean pedicle transaction (A) and the Pringle maneuver (B): occluding the Glissonean pedicle of the left liver lobe (a), the right liver lobe (b), and the right posterior lobe (c); and occluding the liver pedicle.

\section{Postoperative management}

Following surgery, all patients were transferred to the surgical intensive care unit (ICU) for postoperative recovery and monitoring. Discharge from the ICU was based on each patient's postoperative progress and was at the discretion of an independent attending ICU physician, who was blinded to the vascular control technique. Follow-up liver function tests were repeated on days 1,5 , and 10 following surgery. If ascites was suspected, diuretics were administered intravenously along with albumin supplementation, and abdominal ultrasonography or computed tomography was used to monitor the resolution of ascites. Patients were discharged from the hospital in the absence of any clinically significant events, and were followed up at 3-month intervals during the first year and at 6-month intervals there- 
after. Follow-up tests included routine hematological, biochemical, and serological tests as well as abdominal CT scans.

\section{Outcome measures}

The primary outcome measure was postoperative change in liver function from baseline. The secondary outcomes included operating time, volume of intraoperative blood loss, time to resolution of ascites, tumor recurrence, and overall survival.

\section{Statistical analysis}

Numerical data, expressed as mean $\pm S D$, were compared in the two groups using Student- $t$ tests or one-way repeated measures ANOVA. Categorical data were expressed as $\mathrm{n}(\%)$ and were compared using Fisher's exact probability tests. A P-value less than 0.05 was considered statistically significant.

\section{Results}

\section{Baseline characteristics}

The baseline characteristics of the two groups are shown in Table 1 . The two groups were comparable in age, sex, site and size of the liver tumor, segment or lobe intended to be resected, and liver function reserve $(P>0.05$ each). In all patients, the remnant liver volume was expected to be $>50 \%$ of the total liver volume.

Table I. Baseline characteristics of HCC patients $(n=50)$ scheduled for major hepatectomy.

\begin{tabular}{|c|c|c|c|}
\hline & Glissonean group $(\mathrm{n}=25)$ & Pringle group $(n=25)$ & $P$-value \\
\hline Age, year & $55.0 \pm 12.0$ & $58.0 \pm 9.0$ & 0.305 \\
\hline Sex, M/F & $18 / 7$ & $18 / 7$ & 0.572 \\
\hline \multicolumn{4}{|l|}{ Underlying liver conditions, $\mathrm{n}(\%)$} \\
\hline Chronic hepatitis & $24 / 25(96.0)$ & $23 / 25(92.0)$ & 1.000 \\
\hline Liver cirrhosis & $21 / 25(84.0)$ & $23 / 25(92.0)$ & 0.667 \\
\hline Liver steatosis & $22 / 25(88.0)$ & $21 / 25(84.0)$ & 1.000 \\
\hline Liver function reserve & & & 1.000 \\
\hline Child-Pugh class A & $21 / 25(84.0)$ & $22 / 25(88.0)$ & \\
\hline Child-Pugh class B & $4 / 25(16.0)$ & $3 / 25(12.0)$ & \\
\hline ICG $15, \%$ & & & 1.000 \\
\hline$<10 \%$ & $21 / 25(84.0)$ & $22 / 25(88.0)$ & \\
\hline $10-15 \%$ & $4 / 25(16.0)$ & $3 / 25(12.0)$ & \\
\hline \multicolumn{4}{|l|}{ Laboratory values } \\
\hline Hemoglobin, g/dL & $13.2 \pm 2.2$ & $12.4 \pm 2.4$ & 0.214 \\
\hline Platelet count, $10^{9} / \mathrm{L}$ & $105.2 \pm 39.0$ & $113.2 \pm 37.0$ & 0.447 \\
\hline Albumin, $g / L$ & $41.2 \pm 11.1$ & $43.2 \pm 11.3$ & 0.531 \\
\hline AST, IU/mL & $272.2 \pm 112.2$ & $287.2 \pm 114.2$ & 0.642 \\
\hline$A L T, I U / m L$ & $282.1 \pm 118.1$ & $292.1 \pm 114.1$ & 0.762 \\
\hline Total bilirubin, $\mu \mathrm{mol} / \mathrm{L}$ & $22.1 \pm 4.1$ & $24.1 \pm 4.1$ & 0.091 \\
\hline Prothrombin time, INR & $71.1 \pm 16.2$ & $72.1 \pm 16.2$ & 0.828 \\
\hline Size of tumor, cm & $8.0 \pm 2.1$ & $7.8 \pm 2.2$ & 0.737 \\
\hline Number of nodules, $\mathrm{n}$ & $1.2 \pm 0.3$ & $1.3 \pm 0.2$ & 0.172 \\
\hline Location of tumor, $\mathrm{n}(\%)$ & & & 0.625 \\
\hline Left lobe & $7(28.0)$ & $8(32.0)$ & 1.000 \\
\hline Middle lobe & $10(40.0)$ & $12(48.0)$ & 0.776 \\
\hline Right lobe & $8(32.0)$ & $5(20.0)$ & 0.520 \\
\hline Number of segments involved, n (\%) & & & 0.230 \\
\hline One segment & $15(60.0)$ & $19(76)$ & 0.364 \\
\hline Two segments & $8(32.0)$ & $3(12.0)$ & 0.171 \\
\hline Three segments & $2(8.0)$ & $3(12.0)$ & 1.000 \\
\hline Volume of expected remnant liver, \% & $50.0 \pm 13.0$ & $50.0 \pm 11.0$ & 1.000 \\
\hline Vascular involvement, n (\%) & & & \\
\hline Inferior vena cava & $0 / 25(0.0)$ & $0 / 28(0.0)$ & 1.000 \\
\hline Hepatic veins & $18 / 25(72.0 \%)$ & $21 / 28(75.0)$ & 1.000 \\
\hline Portal vein & $20 / 25(80.0)$ & $22 / 28(78.6)$ & 1.000 \\
\hline
\end{tabular}




\section{Surgical outcomes}

Following peritoneal exploration, all the patients were deemed operable and underwent successful major hepatectomies using the assigned method. No patient in the Glissonean group required conversion to the Pringle maneuver (Figure 1). The surgical outcomes of major hepatectomy are summarized in Table 2. The extent of major hepatectomy was comparable in the Glissonean and Pringle groups $(P=$ 0.832 ), including in patients undergoing right hemihepatectomy $(48.0 \%$ vs. $46.4 \%, P=1.000)$, left hemihepatectomy $(40.0 \%$ vs. $35.7 \%, P=0.7832)$, extended right hemihepatectomy $(8.0 \%$ vs. $7.1 \%, P=1.000)$, and extended left hemihepatectomy $(4.0 \%$ vs. $10.7 \%, P=$ 0.6127). Whereas the Glissonean approach shortened the duration of inflow control (30.0 \pm 12.0 min vs. 45.0 $\pm 13.0 \mathrm{~min}, P<0.001$ ), it failed to significantly reduce the time of liver ischemia caused by pedicular vessel control $(29.0 \pm 11.0 \mathrm{~min} v \mathrm{~s}$. $34.0 \pm 12.0 \mathrm{~min}, P=0.122)$. However, blood loss from hepatectomy was significantly lower in the Glissonean than in the Pringle group $(145.0 \pm 20.0 \mathrm{~mL}$ vs. $298.0 \pm 42.0 \mathrm{~mL}, P<0.001)$. None of the patients undergoing Glissonean resection required any transfusion $(0.0 \pm 0.0 \mathrm{~mL} v s .200 .0 \pm 109.0$ $\mathrm{mL}, P<0.001)$. Postoperative ascites occurred in two of the 25 patients in the Glissonean group $(8.0 \%)$ and in three of 25 in the Pringle group $(12.0 \% ; \mathrm{P}=1.000)$, with approximate depths of $3.0 \mathrm{~cm}$ and $4.0 \mathrm{~cm}$, respectively, on abdominal ultrasonography. The ascites responded well to symptomatic treatment with albumin supplementation and diuretics and resolved within $9.5 \pm 1.2 \mathrm{~d}$ in the Glissonean group and within $15.3 \pm 2.4 \mathrm{~d}$ in the Pringle group $(P<0.001)$.

\section{Postoperative liver function}

Liver function results on days 1,5 , and 10 are shown in Figure 3. The two groups exhibited similar ischemia-associated liver injury following surgery, with comparable serum ALT, AST, total bilirubin, and albumin concentrations and prothrombin time over the first 10 postoperative days $(P>0.05)$. On postoperative day 1 , patients undergoing the Pringle maneuver experienced a significantly higher but transient total bilirubin concentration compared with patients undergoing the Glissonean approach (32.3 \pm 3.2 $\mu \mathrm{mol} / \mathrm{L}$ vs. $20.3 \pm 3.2 \mu \mathrm{mol} / \mathrm{L}, \mathrm{P}<0.001)$.

Table 2. Surgical outcomes of patients undergoing major hepatectomy using Glissonean pedicle transection or the Pringle maneuver.

\begin{tabular}{|c|c|c|c|}
\hline & Glissonean group $(n=25)$ & Pringle group $(n=25)$ & $P$-value \\
\hline Type of resection, $\mathrm{n}(\%)$ & & & 1.000 \\
\hline Right hemihepatectomy & $12(48.0)$ & $12(48.0)$ & 1.000 \\
\hline Left hemihepatectomy & $10(40.0)$ & $10(40.0)$ & 1.000 \\
\hline Extended right hemihepatectomy & $2(8.0)$ & $2(8.0)$ & 1.000 \\
\hline Extended left hemihepatectomy & $1(4.0)$ & $1(4.0)$ & 1.000 \\
\hline Overall operation time, $\min$ & $80.0 \pm 25.0$ & $100.0 \pm 35.0$ & 0.022 \\
\hline Duration of inflow control, min & $30.0 \pm 12.0$ & $45.0 \pm 13.0$ & $<0.001$ \\
\hline Duration of ischemia, min & $29.0 \pm 11.0$ & $34.0 \pm 12.0$ & 0.122 \\
\hline Intraoperative blood loss, $\mathrm{mL}$ & $145.0 \pm 20.0$ & $298.0 \pm 42.0$ & $<0.001$ \\
\hline Volume of transfusion, $\mathrm{mL}$ & $0.0 \pm 0.0$ & $200.0 \pm 109.0$ & $<0.001$ \\
\hline Clean tumor margins, $\mathrm{n}(\%)$ & $25 / 25(100.0)$ & $25 / 25(100.0)$ & 1.000 \\
\hline Tumor staging, n (\%) & & & 0.770 \\
\hline$T_{1}$ & $6 / 25(24.0)$ & $6 / 25(24.0)$ & 1.000 \\
\hline$T_{2}$ & $11 / 25(44.0)$ & $12 / 25(48.0)$ & 1.000 \\
\hline$T_{3}$ & $5 / 25(20.0)$ & $6 / 25(24.0)$ & 1.000 \\
\hline$T_{4}$ & $3 / 25(12.0)$ & $1 / 25(4.0)$ & 0.609 \\
\hline Newly emerging ascites & $2 / 25(8.0)$ & $3 / 25(12.0)$ & 1.000 \\
\hline Time of ascites resolution, day & $9.5 \pm 1.2$ & $15.3 \pm 2.4$ & $<0.001$ \\
\hline Postoperative ICU stay, day & $3.6 \pm 0.8$ & $3.8 \pm 0.7$ & 0.352 \\
\hline Postoperative hospital stay, day & $12.0 \pm 1.5$ & $14.0 \pm 2.1$ & $<0.001$ \\
\hline
\end{tabular}



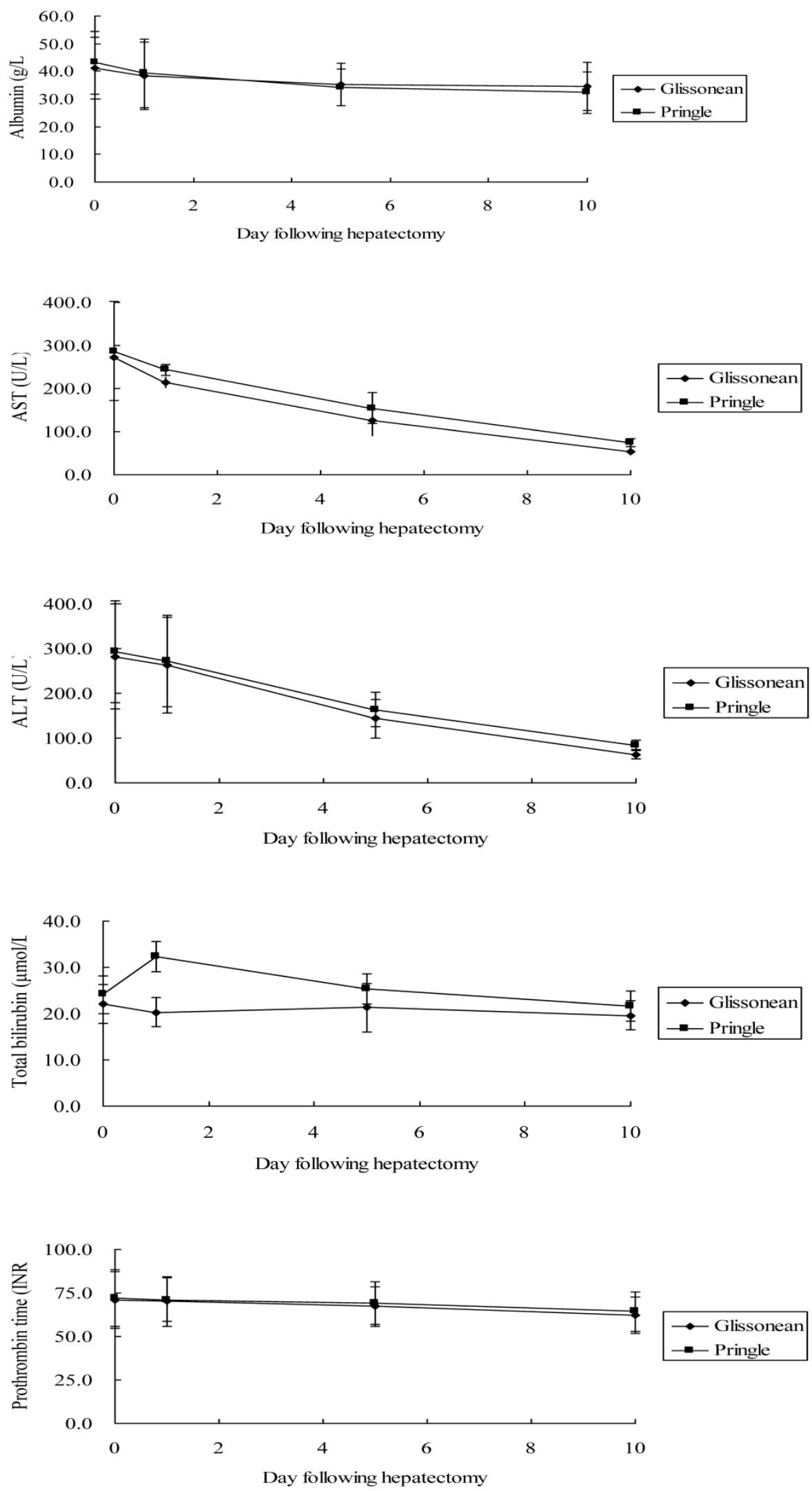

Figure 3. Liver function profiles following major hepatectomy using the Glissonean pedicle transection $(n=25)$ or the Pringle maneuver $(n=25)$ : (A) albumin concentration (reference, 35.0-55.0 g/L); (B) aspartate aminotransferase concentration (reference, < 40U/L); (C) alanine aminotransferase concentration (reference, < $40 \mathrm{U} / \mathrm{L}$ ); (D) total bilirubin concentration (reference, I.7-I7.I $\mu \mathrm{mol} / \mathrm{L}$ ); and (E) prothrombin time (reference, $9.9-12.8$ INR). 


\section{Surgical morbidity and mortality}

Most patients recovered from curative hepatectomy uneventfully regardless of the hilar control approach. The rates of clinically significant surgical morbidities were comparable inbetween the two groups (Table 3), including rates of surgical site infection $(8.0 \%$ vs. $8.0 \%, P=1.000)$, peritoneal bleeding ( $0.0 \%$ vs. $8.0 \%, P=0.490)$, and postoperative pneumonia $(8.0 \%$ vs. $12.0 \%, P=1.000)$. All these complications resolved with routine symptomatic treatment, requiring no secondary surgical intervention. No patient in either group died during hospitalization.

\section{Follow-up and survival outcomes}

The patients were followed up for a mean $36.0 \pm$
5.6 months, with none lost to follow-up through the writing of this manuscript. The cumulative intrahepatic recurrence rates for survivors in the Glissonean and Pringle groups were $8.0 \%$ vs. $10.7 \%(P=1.000)$ at one year, $24.0 \%$ vs. $28.6 \%(P=0.763)$ at two years, and $48.0 \%$ vs. $53.6 \%(P=0.786)$ at three years. Intrahepatic recurrences were controlled using transcatheter arterial chemoembolization, with or without percutaneous radiofrequency ablation. The one- and three-year overall survival rates in the Glissonean and Pringle groups were $42.3 \%$ vs. $32.5 \%(P=1.000)$ and $28.0 \%$ vs. $28.6 \%(P=1.000)$, respectively (Fig. $4 \mathrm{~A})$, and the oneand three-year disease-free survival rates were $72.0 \%$ vs. $64.0 \%(\mathrm{P}=1.000)$ and $4.0 \%$ vs. $4.0 \%(\mathrm{P}=1.000)$, respectively (Fig. 4B).

Table 3. Surgical morbidities and complications in patients undergoing major hepatectomy via Glissonean pedicle transection or the Pringle maneuver.

\begin{tabular}{llll}
\hline & Glissonean group $(\mathrm{n}=25)$ & Pringle group $(\mathrm{n}=25)$ & $P$-value \\
\hline Surgical site infections, $\mathrm{n}(\%)$ & $2 / 25(8.0)$ & $2 / 25(8.0)$ & 1.000 \\
Peritoneal bleeding, $\mathrm{n}(\%)$ & $0 / 25(0.0)$ & $2 / 25(8.0)$ & 0.490 \\
Postoperative pneumonia, $\mathrm{n}(\%)$ & $2 / 25(8.0)$ & $3 / 25(12.0)$ & 1.000 \\
\hline
\end{tabular}
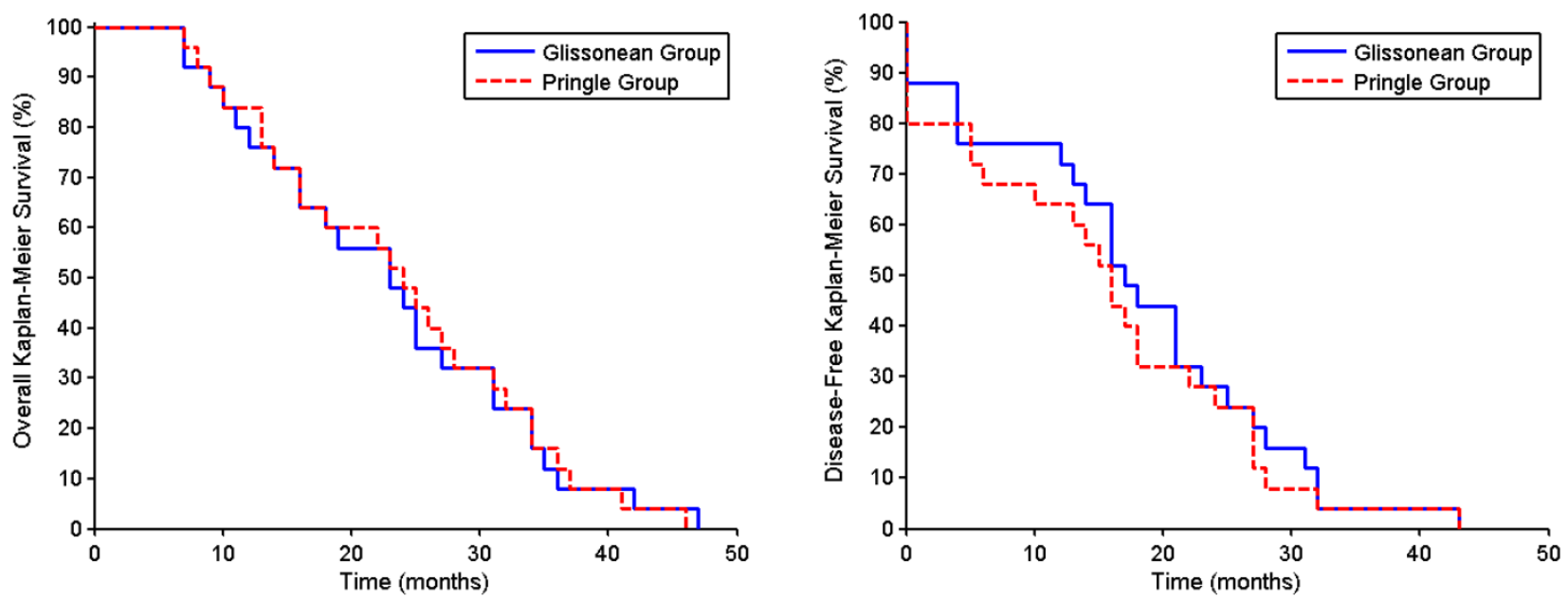

Figure 4. Overall survival rates $(A)$ and disease-free survival rates $(B)$ of HCC patients undergoing major hepatectomy using the Glissonean pedicle transection $(n=25)$ or the Pringle maneuver $(n=25)$.

\section{Discussion}

The Glissonean pedicle transection method has been reported to shorten operation time, reduce intraoperative blood loss, and minimize surgical risks in HCC patients undergoing hepatectomy ${ }^{22}$. The Glissonean approach may therefore be more beneficial for
HCC patients undergoing curative resection than other hilar vascular control methods such as the Pringle maneuver. However, in a large series, only $17.1 \%$ of patients underwent major hepatectomy involving two or more segments, with most patients $(80.3 \%)$ undergoing segmentectomy or subsegmentectomy ${ }^{18}$. Our patient cohort differs in the extent 
of liver resection as well as including only patients with large $(>5 \mathrm{~cm})$ nodular HCCs. $56 \%$ Of the 25 patients in our Glissonean group, 14 (56\%) underwent removal of more than 2.5 segments (right trisegmetectomy).

To our best knowledge, our study is the first to compare the surgical effectiveness and safety of Glissonean transection with the Pringle maneuver in patients with large HCC undergoing radical hepatectomy. We found that the Glissonean approach was feasible, effective, and safe for patients with large HCC and underlying liver conditions. This method yielded better recovery of liver function recovery following major hepatectomy than the conventional Pringle maneuver.

Although the Glissonean method is deemed advantageous over other hilar control methods such as the Pringle maneuver and hilar dissection, it has not been fully investigated in clinical studies, especially in head-to-head comparisons. Even some studies revealed that there were some disadvantages due to this method ${ }^{23}$. Our results are in conflict with these findings, probably due to differences in patient populations (liver metastases versus primary HCC), concomitant liver condition (steatosis versus cirrhosis), and technical modifications (combining Pringle maneuver versus combining partial IVC interruption). Another modification that may have contributed to the favorable outcomes in our study was our use of the "curettage and aspiration dissection technique (CADT)' ${ }^{24}$, which is regarded as a valuable modality for segmentectomy and major hepatectomy resulting in little or no postoperative bleeding. The incorporation of this method into hilar plate dissection allowed the extra-Glisson's capsule en bloc interruption of the hepatic artery and portal vein branches flowing into the segment or lobe to be resected, rather than the individual ligation of the hepatic artery, portal vein and biliary tract. The en bloc interruption of Glisson's pedicle shortened the duration and extent of ischemia during the process of pedicle control, minimizing the adverse effects of ischemia-reperfusion injury on the patient's liver function, especially in patients with complicating steatosis, fibrosis, or cirrhosis. In addition, the Glissonean method reduced intraoperative blood loss, an independent risk factor for postoperative morbidity/mortality and survival in patients undergoing major hepatectomy ${ }^{8}$.

Following the control of the hilum or Glisson's pedicle, oozing from the transected liver is derived mainly from the backflow of the hepatic veins. Although interruption of the hepatic veins has been used to further reduce blood loss from liver transection 25 , interruption of hepatic outflow has been found to increase bleeding from the hepatectomy. Therefore, the patency of hepatic outflow is required for safe liver resection. CVP control is an effective and safe alternative to decrease backflow-associated hemorrhage ${ }^{26}$. The methods most commonly used to control CVP include the restriction of fluid infusion and the use of diuretics and/or nitroglycerin. However, these methods disturb hemodynamics and have an adverse impact on a patient's circulatory and renal functions. In our modified technique, the infrahepatic IVC is temporarily interrupted to reduce the CVP below 3 $\mathrm{cmH}_{2} \mathrm{O}$ and the resultant backflow from the retroheaptic IVC. This technique maximizes the control of hepatic vein bleeding, while having minimal effect on systemic circulation and blood electrolyte profiles. Partial interruption of the infrahepatic IVC may also be tolerated by some elderly patients.

The Glissonean method has also been associated with procedural complications and morbidities 27. Dissection of the liver plate requires hepatotomy in the absence of hepatic inflow control and may cause some bleeding, although the volume is small in most patients. Dissection of the hepatoduodenal ligament may also result in iatrogenic injury to the portal vein branches flowing into the caudate lobe. In patients with biliary anatomic variations, accidental ligation of the biliary confluence can result in obstructive jaundice during early postoperative stages. Although the Glissonean approach has been reported oncologically superior to the Pringle maneuver, due to the minimized likelihood of intrahepatic metastases, our follow-up results showed that the two groups had comparable intrahepatic recurrence and survival rates. This may have been due to the more advanced tumor stage of our patients, who had larger tumors (> $5 \mathrm{~cm})$ and more frequent vascular invasion $(>80 \%)$ as well as prevailing background cirrhosis. Advanced tumor stage has been associated with relatively poor survival $(<30 \%$ at 3 -year vs. $>30 \%$ at 5 -year). These pathological and oncological characteristics of Chinese HCC patients are due to endemic hepatitis B infection.

\section{Conclusion}

The Glissonean pedicle transection method is a feasible, effective, and safe technique for hepatic inflow control during the curative resection of patients with large nodular HCCs. This method shortened the duration of ischemia and reduced the extent of liver parenchyme subjected to ischemia-reperfusion injury, compared with the conventional Pringle maneuver. The Glissonean method also reduced the volume of intraoperative blood loss, enhancing postoperative recovery of liver function. As an anatomical form of 
hepatectomy, the Glissonean method may minimize the occurrence of intrahepatic metastases, contributing to patient survival. A long-time survival follow-up study is ongoing in our institution to determine the effects on oncologic safety and survival of the Glissonean pedicle transection method in patients with large HCCs with complicating liver conditions who undergo major hepatectomy.

\section{Competing Interests}

The authors have declared that no competing interest exists.

\section{References}

1. Tanaka M, Katayama F, Kato H, et al. Hepatitis B and C virus infection and hepatocellular carcinoma in China: a review of epidemiology and control measures. J Epidemiol.2011; 21: 401-16.

2. Tang Z, Zhou X, Lin Z, et al. Surgical treatment of hepatocellular carcinoma and related basic research with special reference to recurrence and metastasis. Chin Med J (Engl).1999;112: 887-91.

3. Livraghi $\mathrm{T}$, Mäkisalo $\mathrm{H}$, Line PD. Treatment options in hepatocellular carcinoma today. Scand J Surg. 2011; 100: 22-9.

4. Takayama T. Surgical treatment for hepatocellular carcinoma. Jpn J Clin Oncol. 2011;41: 447-54.

5. Kudo M. Early detection and curative treatment of early-stage hepatocellular carcinoma. Clin Gastroenterol Hepatol. 2005; 3: S144-8.

6. Matsumata T, Kanematsu T, Shirabe K, et al. Decreased morbidity and mortality rates in surgical patients with hepatocellular carcinoma. Br J Surg. 1990; 77: 677-80.

7. Takenaka K, Kanematsu T, Fukuzawa K, et al. Can hepatic failure after surgery for hepatocellular carcinoma in cirrhotic patients be prevented? World J Surg. 1990; 14: 123-7.

8. Yang T, Zhang J, Lu JH, et al. Risk factors influencing postoperative outcomes of major hepatic resection of hepatocellular carcinoma for patients with underlying liver diseases. World J Surg. 2011; 35: 2073-82.

9. Wang CC, Iyer SG, Low JK, et al. Perioperative factors affecting long-term outcomes of 473 consecutive patients undergoing hepatectomy for hepatocellular carcinoma. Ann Surg Oncol. 2009; 16: 1832-42.

10. Yamashita Y, Taketomi A, Shirabe K, et al. Outcomes of hepatic resection for huge hepatocellular carcinoma ( $\geq 10 \mathrm{~cm}$ in diameter). J Surg Oncol. 2011; 104: 292-8.

11. Fan ST, Lo CM, Poon RT, et al. Continuous improvement of survival outcomes of resection of hepatocellular carcinoma: a 20-year experience. Ann Surg. 2011; 253: 745-58.

12. Chau GY, Lui WY, King KL, Wu CW. Evaluation of effect of hemihepatic vascular occlusion and the Pringle maneuver during hepatic resection for patients with hepatocellular carcinoma and impaired liver function. World J Surg. 2005; 29: 1374-83.

13. Hatipoglu AR, Temiz E, Yüksel $M$, et al. The comparison of electron microscopy and scintigraphy in determining the protective effect of dimethylsulphoxide (DMSO) on ischemia/reperfusion injury through Pringle maneuver. Hepatogastroenterology. 2001; 48: 799-802.

14. Ypsilantis P, Lambropoulou M, Anagnostopoulos C, et al. Pringle maneuver exacerbates systemic inflammatory response and multiple-organ injury induced by extended liver radiofrequency ablation. Hum Exp Toxicol. 2011; 30: 1855-64.

15. Zhou W, Li A, Pan Z, et al. Selective hepatic vascular exclusion and Pringle maneuver: a comparative study in liver resection. Eur J Surg Oncol. 2008; 34: 49-54.

16. Horgan PG, Leen E. A simple technique for vascular control during hepatectomy: the half-Pringle. Am J Surg. 2001; 182: 265-7.

17. Kukita K, Katsuramaki T, Kikuchi H, et al. Remnant liver injury after hepatectomy with the pringle maneuver and its inhibition by an iNOS inhibitor (ONO-1714) in a pig model. J Surg Res. 2005; 125: 78-87.

18. Takasaki K. Glissonean pedicle transection method for hepatic resection: a new concept of liver segmentation. J Hepatobiliary Pancreat Surg. 1998; 5: 286-91.

19. Cho A, Asano T, Yamamoto H, et al. Laparoscopy-assisted hepatic lobectomy using hilar Glissonean pedicle transection. Surg Endosc. 2007; 21: $1466-8$.
20. Wu CC, Hsieh SR, Chen JT, et al. An appraisal of liver and portal vein resection for hepatocellular carcinoma with tumor thrombi extending to portal bifurcation. Arch Surg. 2000;135:1273-9.

21. Fu SY, Lau WY, Li GG, et al. A prospective randomized controlled trial to compare Pringle maneuver, hemihepatic vascular inflow occlusion, and main portal vein inflow occlusion in partial hepatectomy. Am J Surg. 2011; 201: 62-9.

22. Yamamoto M, Takasaki K, Ohtsubo T, et al. Effectiveness of systematized hepatectomy with Glisson's pedicle transection at the hepatic hilus for small nodular hepatocellular carcinoma: retrospective analysis. Surgery. 2001; 130: 443-8.

23. Hardy KJ, Jones RM. Failure of the portal vein to bifurcate. Surgery. 1997;121:226-8.

24. Peng SY, Li JT, Mou YP, et al. Different approaches to caudate lobectomy with "curettage and aspiration" technique using a special instrument PMOD: a report of 76 cases. World J Gastroenterol. 2003; 9: 2169-73.

25. Berney T, Mentha G, Morel P. Total vascular exclusion of the liver for the resection of lesions in contact with the vena cava or the hepatic veins. $\mathrm{Br} \mathrm{J}$ Surg. 1998; 85: 485-8.

26. Wang WD, Liang $L J$, Huang $X Q$, et al. Low central venous pressure reduces blood loss in hepatectomy. World J Gastroenterol. 2006; 12: 935-9.

27. Fujii M, Shimada M, Satoru I, et al. A standardized safe hepatectomy; selective Glissonean transection using endolinear stapling devices. Hepatogastroenterology. 2007; 54: 906-9. 